\title{
Mechanical Characterization of Micro/Nano Structures
}

\author{
Daniel Kujawski* and Muralidhar K. Ghantasala
}

Department of Mechanical and Aeronautical Engineering, Western Michigan University, Kalamazoo, MI, USA

\begin{abstract}
This paper reviews some of the important characterization techniques used to evaluate the mechanical properties of the micro or nanostructures and components. Three techniques based on AFM, nanoindentation and electric field induced resonance excitation methods were discussed in detail. In the following section mechanical characteristics of typical semiconducting or metallic materials with an emphasis on their micro/nano components are discussed. The influence of cross-section dimensions on the quasi-static strength of single and poly crystalline silicon micro specimens in bending and tension is examined. The differences in mechanical properties, testing methods and specimen dimensions were analyzed based on the recently reported data. A simple empirical correlation is presented with interpretation of the experimental data.
\end{abstract}

Keywords: Micro/nano materials, strength, tension, bending, fatigue, nanoindentation.

\section{INTRODUCTION}

The advent of micromechanical structures and the recent trends of a transition from micro to nano systems require new tools and techniques for their mechanical characterization. Ever increasing surface to volume ratios of continuously decreasing sizes of these structures into the nano world has impacted the traditional modeling and mathematical treatment approaches. This situation has further been complicated by the use of different material forms (e.g., nanotubes, nanowires, nanoporous structures etc.), their processing technologies and more importantly presence of many such materials in one structure. All these aspects additionally complicate the characterization methodologies to another level from already complex problems. It was observed by different researchers that the properties of materials such as Young's modulus, yield and ultimate strength, ductility, toughness, crack initiation and growth change for the same material from bulk form down to devices with nano/micro dimensions. The unique properties of carbon nanotubes and the likes will have to be characterized carefully, when layers of nanotubes and/or nanowires are part of multi-layered structures. As the exact dimension, where the size effects become predominant causing different properties deviate from their bulk characteristics, is also not clear, it leads to a grey area in terms of application of a specific characterization technology. It is obvious that stress-strain relationship in thin films is affected by the relatively high area to volume ratio. As a result, typical properties used for description of bulk material strength and deformation do not apply for thin films and micro devices. In particular, this size effect relationship is not yet well understood when structural dimensions decrease from millimeters to micro and nanometers.

\section{TESTING METHODS}

During the late 1970s and 1980s the basic mechanical properties were usually determined using simple testing methods such as frequency resonance [1], beam bending [2],

*Address correspondence to this author at the Department of Mechanical and Aeronautical Engineering, Western Michigan University, Kalamazoo, MI, USA; E-mail: daniel.kujawski@wmich.edu and membrane-bulge [3] tests. The rapid advances in MEMS and the recent development of nano tubes, wires, cantilevers, belts, springs and other one dimensional (1D) nanostructures necessitated improvements in characterization tools as well as methods, to obtain reliable mechanical data especially in order to understand the effects of sample sizes (in micro and nano regime) and used fabrication processes.

Mechanical characterization of 1D nano-components is still a very nascent field as the testing methods are not well developed yet. This has further been complicated by the lack of appropriate analytical tools and models involved in the interpretation of the experimental data obtained at the molecular level. One of the main problems arises from the fact that the techniques used at the macro level cannot be implemented at the micro and nano levels. For example, tensile testing requires a rigid clamping with a good alignment that is not easy to implement at the nano-level. Even simple positioning and manipulation of nanostructures or parts is difficult. Among the characterization techniques commonly used for thin films and micro/nanostructures are: atomic force microscopy (AFM), nanoindentation, and resonance based mechanical testing. Most of these methods involve the use of MEMS test platforms to mount the test structures. In the present context, we will discuss these techniques in a reasonable detail.

\section{AFM Characterization}

AFM based technique, due to its high spatial resolution and also force and displacement sensing capabilities, has been extensively used for the measurement of mechanical properties of the nano/micro structured elements. Elastic modulus determination as well as fatigue testing of a chosen micro-component can be carried out using the set-up shown in Fig. (1).

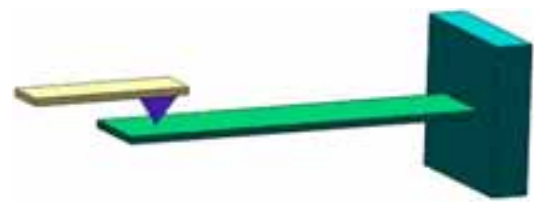

Fig. (1). Typical experimental setup showing the relative positions of the micro-cantilever and AFM tip. 
This method is commonly used in bending configuration. The displacement of the micro-cantilever under a point (normal) load is given by [4-6].

$\mathrm{D}_{\text {micro-cantilever }}=\mathrm{D}_{\text {piezo }}-\mathrm{D}_{\text {tip }}$

where $\mathrm{D}_{\text {piezo }}$ is the piezoactuator based movement in the vertical direction, $\mathrm{D}_{\text {tip }}$ corresponds to the tip deflection determined using the force calibration mode. Piezo actuator is commonly used in AFM to facilitate movement of the cantilever.

Normal load on the cantilever $\left(\mathrm{W}_{\text {micro-cantilever }}\right)$ is same as the normal load on the AFM tip ( $\mathrm{W}_{\text {tip }}$ ) and is given by:

$\mathrm{W}_{\text {micro-cantilever }}=\mathrm{W}_{\text {tip }}=\mathrm{kD}_{\text {tip }}$

where $\mathrm{k}$ is the spring constant of the AFM cantilever. This provides a load-displacement curve for the material of interest. Slope of the load-displacement curve provides the stiffness of the microcantilver being tested.

$\mathrm{S}=\Delta \mathrm{W}_{\text {micro-cantilever }} / \Delta \mathrm{D}_{\text {micro-cantilever }}$

Knowing $\mathrm{W}_{\text {micro-cantilever }}$ and $\mathrm{D}$ micro-cantilever, elastic modulus can also be estimated using the following relationship,

$\mathrm{E}=1^{3} * \mathrm{~W}_{\text {microcantilever }} / 3 \mathrm{I} \mathrm{D}_{\text {micro-cantilever }}$

where $\mathrm{I}=(1 / 12) * \mathrm{w}^{*} \mathrm{t}^{3}$ is the area moment of inertia, $\mathrm{w}=$ width, $\mathrm{t}=$ thickness, and 1 being the length of the microcantilever.

Maximum bending stress at the fixed end of the microcantilever is calculated using standard relations [5]. In order to study the fatigue characteristics, mean loads are applied on the free end of the micro-cantilever using AFM tip. A known cyclic load of $\mathrm{W}_{\mathrm{a}}$ is applied on the micro-cantilever with required frequency (For example, 10 to $20 \mathrm{nN}$ at a frequency of around $4 \mathrm{~Hz}$ used in a study by Liu and Bhushan [5]). The actual load, tip deflection and the vertical-piezo movement were continuously monitored. A sudden drop of micro-cantilever stiffness is considered as an indicator of its failure. S-N curves obtained showed a similar failure mode commonly observed with thin film samples. Liu and Bhushan reported that at a load of $12 \mathrm{nN}$, the cycles to failure were $10^{4}$, which were found to increase to $10^{5}$ cycles at a load of $10 \mathrm{nN}$ in their study of fatigue characteristics of a hinge used in a MEMS based digital mirror device [5].

Results on the cyclic bending tests on nanoscale single and poly crystalline silicon wires using AFM [7, 8] will be discussed in a later section.

\section{Nanoindentation}

Nanoindentation is one of the most effective tools for the study of mechanical properties of micro or 1D nanomaterials used in various applications. In order to determine the mechanical properties of micro (cantilever or bridge) or nano (wires, tubes or belts) samples are placed between two stable edge structures fabricated using standard microfabrication methods. Nanoindenter tip is moved over to the chosen location of the sample, where the concerned properties are to be determined. Nanoindenter monitors the load and displacement of the indenter during indentation with a force resolution of $1 \mathrm{nN}$ or less with a displacement resolution of $2 \mathrm{~A}$, the absolute values of which depend on the instrument. Three sided pyramidal diamond (Berkovitch) is used as the inden- ter, which provides relatively simpler analysis using the methods of continuum mechanics [9].

During a nanoindentation test, force and displacement values are recorded as the indenter tip is pressed into the test material's surface. Typical load versus displacement curve often seen in a nanoindentation experiment is shown in Fig. (2). It may be noted that the shape and scales will be different for different materials and experiments.

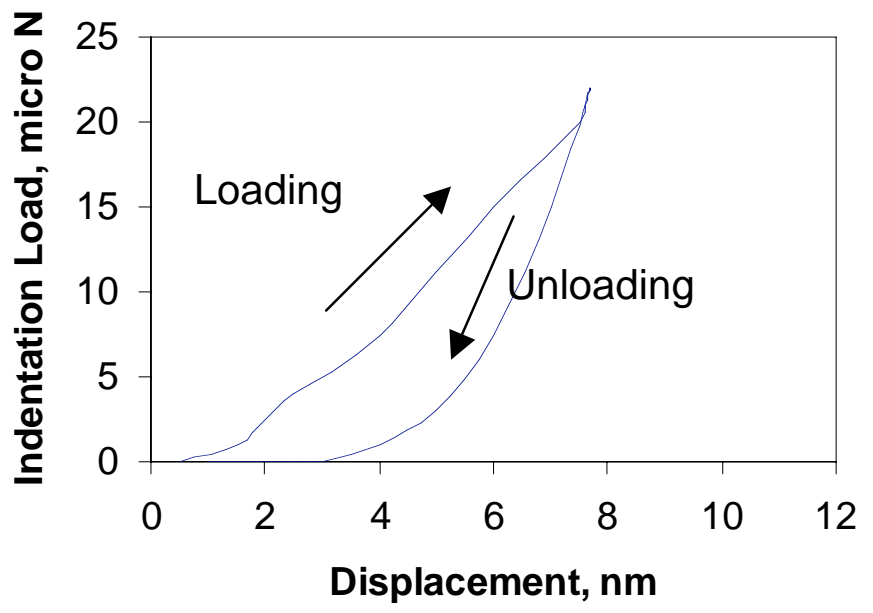

Fig. (2). Typical nanoindentation load $v s$ displacement curve.

These load-displacement curves, not only provide the extent of plastic deformation due to the loading-unloading cycles, but also exhibit localized perturbations or discontinuities, which are represented by the characteristic signatures of energy absorbing or releasing events occurring beneath the tip. In an investigation by $\mathrm{Ni}$ and $\mathrm{Li}$, it was shown that $\mathrm{ZnO}$ nanobelts failed from brittle fracture in bending, while indicated some plastic deformation in nanoindentation [6]. The load-displacement curves obtained in that study through nanoindentation showed a permanent deformation approximately at around $3 \mathrm{~nm}$, after the peak indentation load was removed from $\mathrm{ZnO}$ nanobelts with a thickness of $95 \mathrm{~nm}$ and a width of $800 \mathrm{~nm}$.

Young's Modulus $\left(\mathrm{E}_{\mathrm{n}}\right)$ can be determined following the linear elastic theory of an isotropic material, knowing the spring constant of the structure to be tested using the formula,

$\mathrm{E}_{\mathrm{n}}=\mathrm{FL}^{3} / 192 \mathrm{~d}_{\mathrm{n}} \mathrm{I}$

where $\mathrm{F}$ is the force, $\mathrm{L}$ is the suspended length of the structure or a micro/nano bridge, $\left(\mathrm{F} / \mathrm{d}_{\mathrm{n}}\right)$ - slope of the forcedisplacement curve during bending represents the spring constant $\left(K_{n}\right)$ and $I$ is the area moment of inertia.

Nanoindentation hardness is defined as the indentation load divided by the projected contact area of the indentation, as defined by the formula $[10,11]$

$\mathrm{H}=\mathrm{P}_{\max } / \mathrm{A}$

$\mathrm{P}_{\max }$ is the peak load, while $\mathrm{A}=(\pi / 4)\left(\mathrm{S} / \mathrm{E}_{\mathrm{r}}\right)^{2}$, is the projected contact area.

$\mathrm{E}_{\mathrm{r}}$ - reduced elastic modulus and $\mathrm{S}=(\mathrm{dP} / \mathrm{dH})$-slope of the initial portion of the unloading curve.

$\left(1 / \mathrm{E}_{\mathrm{r}}\right)=\left[\left(1-\mathrm{v}_{\mathrm{s}}^{2}\right) / \mathrm{E}_{\mathrm{s}}\right]+\left[\left(1-\mathrm{v}_{\mathrm{i}}^{2}\right) / \mathrm{E}_{\mathrm{i}}\right]$ 
where $E_{\mathrm{s}}, \mathrm{E}_{\mathrm{i}}$ and $v_{\mathrm{s}}, v_{\mathrm{i}}$ are the young modulie and Poissons' ratio of the sample and indenter respectively. It may be noted that the subscript $s$ and $i$ refer to the substrate and indenter, respectively. The bending stresses generated in the micro bridge (or nano wire) are proportional to the moments generated at the fixed ends. The maximum tensile stress, $\sigma_{b}$, which is the fracture stress, is given by Li and Bhushan [12]

$\sigma_{\mathrm{b}}=\left(\mathrm{F}_{\max } \mathrm{le}_{1} / 8 \mathrm{I}\right)$

where $F_{\max }$ is the load applied at the instant of failure, 1 is the length of the beam, and $e_{1}$ is the distance of the top surface from the neutral plane of its cross-section.

\section{Electric Field Induced Resonance Excitation Method}

In this method, electric field induced resonance excitation is used for measuring different mechanical properties of individual nanowire like structures inside a TEM (Transmission Electron Microscope). This technique has been successfully used to study the properties of carbon nanotubes, silicon nanowires, $\mathrm{SiC}-\mathrm{SiO}_{2}$ composite nanowires and $\mathrm{ZnO}$ nanobelts. The specimen holder used in these measurements has two electrodes and a set of peizomanipulation and translation stages. Typical electrical measurement setup shown in Fig. (3). is a simple schematic only, a more detailed representation of the set-up with an in situ TEM measurement arrangement can be seen in a paper by Wang et al [13].

Different voltage configurations were used to determine the bending modulus of the individual nanotubes, wires and belts. From classical elasticity theory, the fundamental resonance frequency is related to the bending modulus and other nanodimensional parameters by

$v_{i}=\frac{\beta_{i}^{2}}{8 \pi L^{2}} \sqrt{\frac{\left(D^{2}+D_{i}^{2}\right) E_{\beta}}{\rho}}$

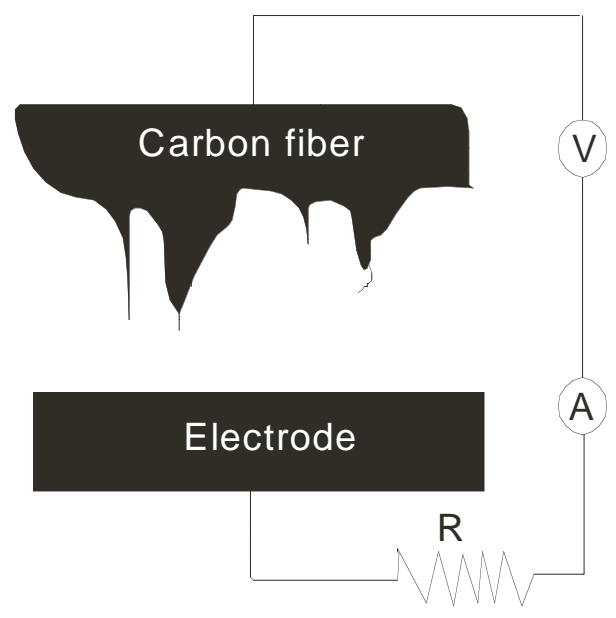

Fig. (3). An experimental setup used during the electric field induced resonance excitation method [13].

where $v_{i}$ is the resonance frequency, $\beta_{i}$ is a constant for $i$ th harmonic. $E_{\beta}$ is the bending modulus, $\rho$ density of the tube, $\mathrm{L}, \mathrm{D}$ and $\mathrm{D}_{\mathrm{i}}$ are the length, outer and inner diameter of the tube respectively. Same relationship can be extended to nanowires or belts representing the diameter of the wire or width of the belt.

Individual nanocomponent is attached to both the gold electrodes using silver paste, through which the electric con- tacts were made. A constant or alternating voltage is applied to the two electrodes to induce electrostatic deflection or mechanical resonance.

As these tests are performed in TEM, physical observation of changes of the nanoelement features in response to dynamically changing electrical parameters, has been an added advantage of this method. However, TEM may possibly be replaced by other imaging instruments, which can provide the same information. Due to the electric charge induced at the tip of the 1D nanomaterials, these oscillate at the frequency of the applied voltage. Mechanical resonance results when the applied frequency matches the natural vibration frequency. One needs to determine the resonance frequency accurately, in order to obtain the bending modulus reliably. Authors have extended the same technique for determining the bending modulus of $\mathrm{ZnO}$ nanobelts, Single and double walled carbon nanotubes and other nanowire systems [14-16].

In case of nanobelts which have a rectangular crosssection, two fundamental resonance modes corresponding to two orthogonal transverse vibration directions were observed [14]. Bending modulus of $\mathrm{ZnO}$ nanobelts was measured to be $52 \mathrm{GPa}$, which is much lower than the bulk $\mathrm{ZnO}$ value, which lies in the range 104 to $210 \mathrm{GPa}$. This difference in observed value for single nanobelts was attributed to the scaling effect (with respect to size) and geometrical shape. Further, the anisotropic nature of $\mathrm{ZnO}$ may also be another reason showing large differences even in bulk in different directions. This has been one of the very unique techniques, especially measuring the individual single nanotube/wire/belts. Interestingly, these nanowires and belts may be considered similar to nanocantilevers. The values obtained by this technique were in reasonable agreement with those obtained by a nanoindentor [6]. This technique may potentially be extended for determining the fatigue characteristics of the individual 1D nanomaterial components.

Most of the above mentioned techniques used MEMS based platforms for studying the mechanical properties of the micro or nanostructures in the form of bridges or cantilevers. Measurements were performed using AFM or in situ TEM. The biggest challenge is mounting or fixing the nanosamples on to the MEMS platforms. Few research groups have been successful in designing and developing the MEMS test beds for mechanical characterization of nanowires, belts etc $[17,18]$.

\section{SILICON: SELECTED TEST RESULTS}

Silicon in single or polycrystalline form (or as a compound e.g., $\mathrm{SiO}_{2}, \mathrm{Si}_{3} \mathrm{~N}_{4} \ldots$ ) which had not previously been considered as engineering material is by far the most commonly used mechanical material in MEMS technology today. The most attractive feature of silicon for these applications is that it facilitates ease of fabrication of micromechanical elements using well established micromachining technologies at the same time enabling the integration of these elements with conventional microelectronic chips and devices. In order to assess the long-term durability of MEMS devices, a fundamental understanding and determination of the strength characteristics of micro-devices in terms of Young's modulus, the fracture strength, ductility, and fatigue performance is required. The testing methods of choice to- 
day seem to be the bending and tension testing $[19,20]$. These testing methods allow for integration of microsamples and miniaturized loading systems, where the applied forces and actual displacements are measured. Further, Mechanical properties of CNTs were also reported to have been measured by stretching or bending between AFM probe tips and were excited using thermal energy [18] while monitoring vibration amplitude in order to observe mechanical strength.

A round robin study [21] demonstrated the inconsistency of measured modulus and strength values, even when specimens from the same source were examined. The samples were fabricated at the Cronos using the same wafer, the same run and the same deposition reactor. It was reported that the modulus and strength values demonstrated rather wide spread, namely from 132 to $174 \mathrm{MPa}$ for Young's moduli whereas the strength varied from 1.0 GPa, for specimen tested in tension, to $2.7 \mathrm{GPa}$ for specimens tested in bending. Subsequently the second round robin conducted on material fabricated at the Sandia National Laboratories [22] also demonstrated a dependence of rapture strengths on specimen size and measurement techniques.

Data from round robin conducted in Japan on the singlecrystal silicon films indicated that Young's modulus apply down to a length of fraction of micrometers. The average value of Young's modulus was about $170 \mathrm{GPa}$ (with standard deviation $10 \%$ of the average) that was in agreement with the theoretical modulus of $168.9 \mathrm{GPa}$ [23]. In contrast, the same round robin [23] demonstrated that tensile strength of polysilicon films determined using on-chip tensile testing device increased from $2.2 \mathrm{GPa}$ to $2.8 \mathrm{GPa}$ with decreasing lengths from $300 \mu \mathrm{m}$ to $30 \mu \mathrm{m}$. The thickness of the polysilicon films was about $2 \mu \mathrm{m}$, the width of the testing gauge was 2 or $5 \mu \mathrm{m}$ and the lengths were 30,100 or $300 \mu \mathrm{m}$. The theoretical analysis of a defect free Si crystal suggests very high strength of $23 \mathrm{GPa}$. On the other hand, at the macroscale the apparent strength is greatly reduced due to existing defects. For example, mm-size samples exhibit an average strength of $0.5 \mathrm{MPa}$ which is only $1 / 50^{\text {th }}$ of the predicted theoretical value [7]. Therefore, if significant variations in the measured properties are accoutered with different sample size and testing methodology, it raises a question on how to account for these effects. This further creates a dilemma on whether the mechanical properties need to be measured with micro samples that are similar in size to the MEMS components or the actual properties may be scaled/inferred from specimens that are relatively large compared to the MEMS structures. It is also not clear with respect to the methods that can be used to compare experimental data obtained at different laboratories using different sample sizes when a fuzzy gap exists between macro and micro scale results.

Fig. (4) shows a general trend of the fracture strength reported using tensile and bending testing of silicon in microscale and nanoscale in terms of stress gradient (Fig. 4a), (cross-section area) ${ }^{0.5}$ (Fig. 4b) and area/volume ratio (Fig. 4c).

Fracture strengths vary remarkably from bulk properties for both tensile and bending data. Bending data show significant increase in strength as stress gradient increases. Both bending and tensile strength increases with decreasing crosssection dimensions or increasing area/volume ratio. It can be
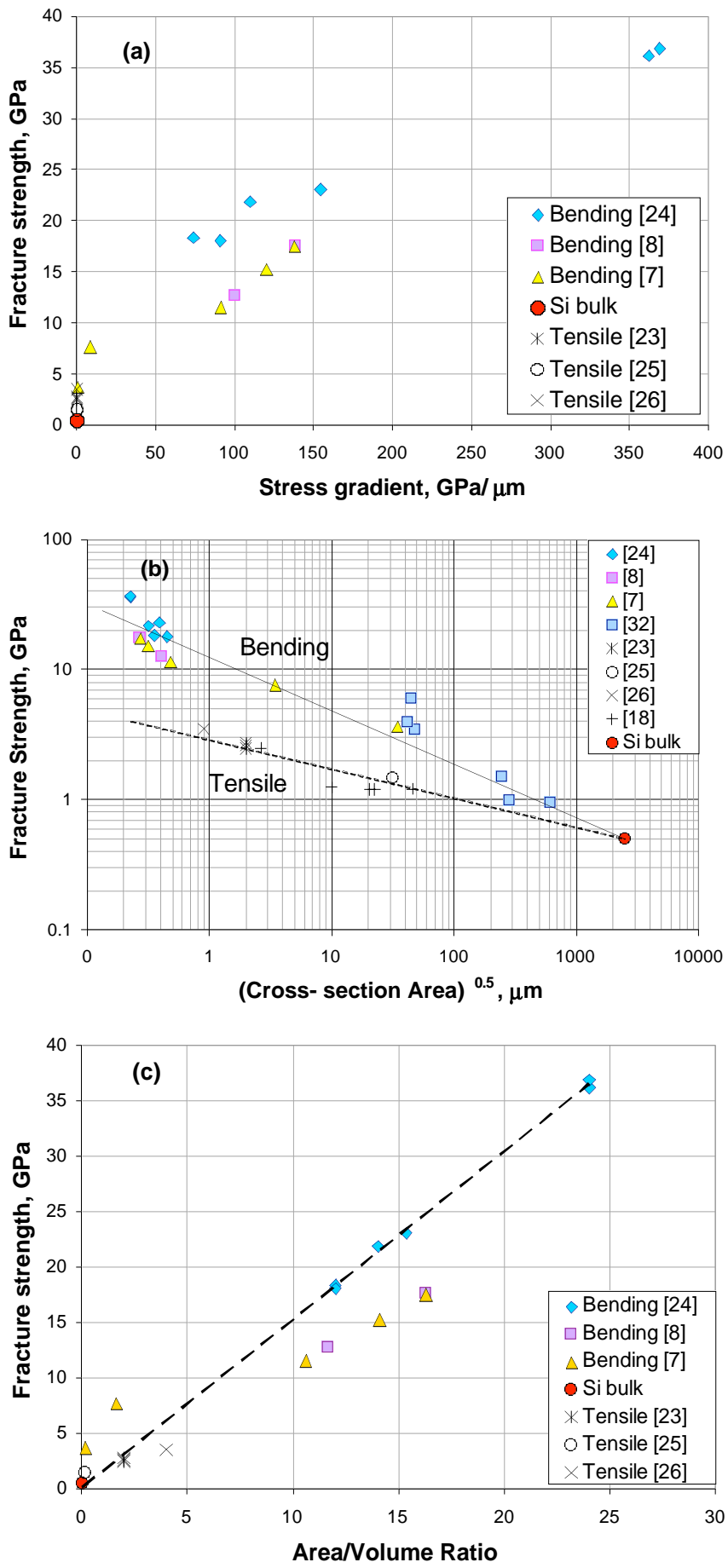

Fig. (4). Bending and tensile strength of $\mathrm{Si}$ nano/micro samples versus (a) stress gradient, (b) (cross-section area) $)^{0.5}$, (c) area/volume ratio.

argued that in brittle Si films, the increased amount of surface energy per unit volume may contribute to the observed increasing strength with increasing area/volume ratio. For bending micro/nano beams there is also high stress gradient which additionally constrain deformation and contribute to even higher strengthening effect in comparison to tensile data. Furthermore, comparison of tensile versus bending data in Fig. (4b) indicates that stress gradient only partially explain the observed size effect. It is noted that (cross-section area) $)^{0.5}$ and area/volume ratio scales the fracture strength 
down to bulk value for both bending and tensile tests (Fig. $4 \mathbf{b}, \mathbf{c})$.

Similar fracture strength data for miniature samples of ductile metallic materials are not very consistent as those for brittle silicon. Fig. (5) depicts data for $\mathrm{Cu}$ and Ni mini samples versus (cross-section area $)^{0.5}$. As the (cross-section area $)^{0.5}$ decreases from $50 \mu \mathrm{m}$ to smaller dimensions $\mathrm{Ni}$ samples exhibit the same trend as silicon, however $\mathrm{Cu}$ samples demonstrated the opposite trend. Both, $\mathrm{Cu}$ and $\mathrm{Ni}$ have similar bulk properties in terms of the ultimate strength and an elongation to fracture.

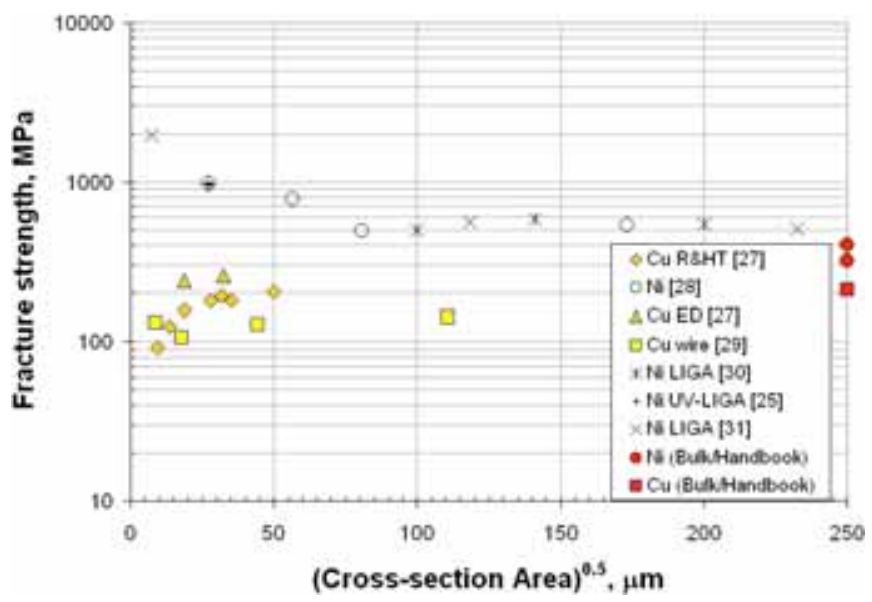

Fig. (5). Fracture strength of $\mathrm{Cu}$ and $\mathrm{Ni}$ micro samples versus (cross-section area) $)^{0.5}$ (R\&HT - rolled and heat-treated, ED - electro-deposited).

\section{Test Results for Other Materials}

In contrast to their strengths, both materials $\mathrm{Cu}$ and $\mathrm{Ni}$ demonstrated decreasing elongation at fracture with decreasing sample (cross-section area) ${ }^{0.5}$ as it is seen in Fig. (6). The reason for this decreasing ductility seems to be associated with non-homogeneity of deformation as grain size approaches sample thickness or width. The effect is likely to be enhanced in grains with preferred orientation which results in strain localization and additional stress concentration. This in turn may lower the material ultimate strength. Therefore the apparent competition between the strengthening effect due to surface energy and weakening effect due to nonhomogeneity of deformation may result in dissimilar behavior as it is indicated in Fig. (5).

It is noted that micro samples made of $\mathrm{Ni}$ demonstrate larger decrease in ductility than $\mathrm{Cu}$ samples (Fig. 6). It is well known that $\mathrm{Ni}$ is more affected by environment than $\mathrm{Cu}$. As a result, $\mathrm{Ni}$ micro samples are more brittle than $\mathrm{Cu}$ micro samples and this is probably the reason why the size effect for Ni data shows the same trend as this for a single and poly crystalline silicon.

\section{SUMMARY}

This paper discussed different mechanical characterization methods for testing micro or nanostructures such as carbon nanotubes, nanowires, nanobelts etc. The published data on monotonic properties of single and poly crystalline silicon show that elastic modulus obtained from miniaturized tensile samples are comparable with those expected for bulk materials. On the other hand, fracture strength may vary signifi- cantly from bulk properties. In particular, bending data show significant increase in strength as thickness or cross-section dimensions decrease. The size effect of increasing strength with decreasing cross-section dimensions of brittle Si miniature samples can be attributed to the increased amount of surface energy per unit volume. For bending test there is also high stress gradient which additionally constraint deformation and contributes to strengthening effect.

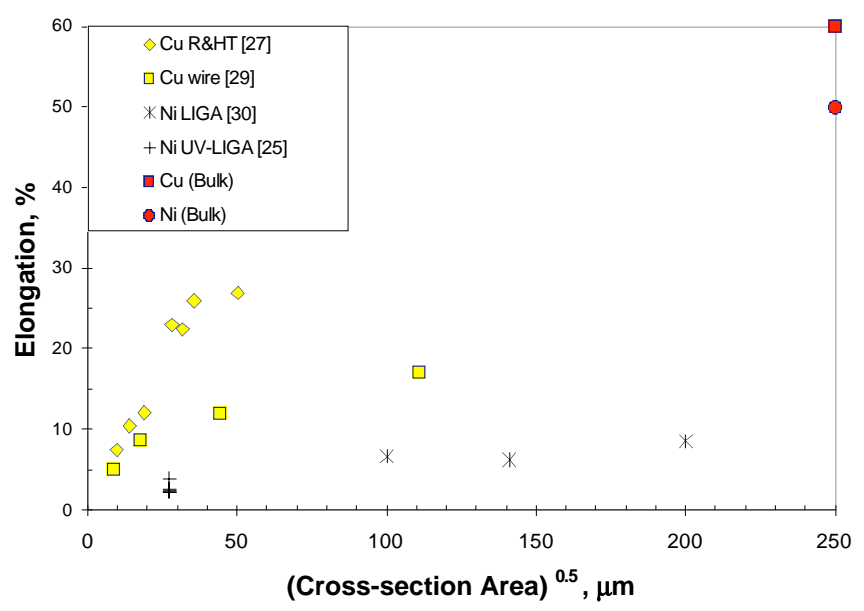

Fig. (6). Elongation to fracture of $\mathrm{Cu}$ and $\mathrm{Ni}$ micro samples versus (cross-section area) $^{0.5}$ (R\&HT - rolled and heat-treated, ED - electro-deposited).

For metallic materials the most commonly observed size effect in miniature samples is a trend of decreasing ductility with decreasing sample thickness and width. The reason is mainly associated with non-homogeneity of deformation as grain size approaches sample thickness or width. The effect is likely to be enhanced in grains with preferred orientation which results in strain localization and additional stress concentration. Ductility may be reduced further with increasing surface topography and environmental effects. Reduced ductility affects the material strength due to higher sensitivity to porosity, inclusions and surface roughness. Therefore, the apparent competition between the strengthening effect due to surface energy/stress gradient and weakening effect due to non-homogeneity of deformation for metallic thin films results in inconclusive data reported in the literature. However, further analysis is required to understand the size effects in relation to processing methods (e.g., electroplating, self assembly and others) and the testing mode (tensile or bending) used.

\section{ACKNOWLEDGEMENTS}

This study is partially supported by the Office of Naval Research under grants N00014-04-1-0718.

\section{REFERENCES}

[1] Petersen, K.; Guarnieri, C. J. Appl. Phys., 1979, 50, 6761.

[2] Johansson, S.; Schweitz, J.A.; Tenerz, L.; Tiren, J. J. Appl. Phys., 1988, 63, 4799.

[3] Walker, J.A.; Gabriel, K.J.; Mehregany, M. Proc. IEEE Micro Electro Mechanical Systems Workshop, Napa Valley, CA, 1990, 56.

[4] Sundararajan, S.; Bhushan, B. Sensors Actuators A, 2002, 101, 338

[5] Liu, H.; Bhushan, B. Nanotechology, 2004, 15, 1246.

[6] Ni, H.; Li, X, Nanotechnology, 2006, 17, 3591.

[7] Namazu, T.; Isono, Y.; Tanaka, T. J. Microelectromech. Sys., 2000, 9,450 . 
[8] Namazu, T.; Isono, Y. IEEE Proc., 2003, 662.

[9] Schuh, C.A. Materials Today, 2006, 9(5), 32.

[10] Bansal, S.; Toimil-Molares, E.; Saxena, A.; Tummala, R.R., Proc. Electron. Components Technol. Conf., $31^{\text {st }}$ May- $3^{\text {rd }}$ June 2005, 1 , 71.

[11] Li, X.; Gao, H.; Murphy, C.J.; Caswell, K.K. Nano Lett., 2003, 3, 1495.

[12] Li, X.; Bhushan, B. Surf. Coat. Technol., 2003, 163-164, 521.

[13] (a) Wang, Z L.; Poncharal, P.; de Heer, W A. J. Phys. Chem. Solids, 2000, 61, 1025. (b) Wang, Z L.; Poncharal, P.; de Heer, W A. Pure Appl. Chem., 2000, 72, 209.

[14] Bai, X.D.; Gao, P.X.; Wang, Z.L.; Wang, E.G. Appl. Phys. Lett., 2003, 82(26), 4806.

[15] Wang, Z.L.; Dai, Z.R.; Bai, Z.G.; Gao, R.P.; Gole, J. Appl. Phys. Lett., 2000, 77, 3349.

[16] Poncharal, P.; Wang, Z.L.; Ugarte, D.; de Heer, W.A. Science, 1999, 283, 1513.

[17] Desai, A.V.; Haque, M.A. Proc. IMech.E. Nanoeng. Nanosys., 2006, 219, 57.

[18] (a) Yi, T.; Kim, C.-J. Measuret Sci. Technol., 1999, 10, 706. (b) Chris-tofer, H. J. Micromech. Microeng., 2004, 14, S1-S11. (c) Yu, M.; Lourie, O.; Dyer, M.J.; Moloni, K.; Kelly, T.F.; Ruoff, R.S. Science, 2000, 287, 637-40. (d) Krishnan, A.; Dujardin, E.; Ebbesen, T. W.; Yianilos, P.N.; Treacy, M.M.J. Phys. Rev. B, 1998, 58, 14013. (e) Salvetat, J.-P.; Bonard, J.-M.; Thomson, N.H.; Kulik, A.J.; Forr`o, L.; Benoit, W.; Zuppiroli, L. Appl. Phys. A, 1999, 69, 255.
[19] Haque, M.A.; Saif, M.T.A. Soc. Exp. Mech., 2003, 43(3), 248-255

[20] Sharpe, W.N. Jr.; Bogdahn, J. Mech. Mater., 2004, 36, 3-11.

[21] Sharpe, W.N., Jr.; Brown, J.S.; Johnson, G.C.; Knauss, W.G. Materials Research Society Proceedings, San Francicso, CA., 1998, 518, 57.

[22] Lavan, D.A.; Tsuchyia, T.; Coles, G.; Knauss, W.G.; Chasiotis, I.; Read, D.; Muhlstain, C.; Brown, SB., (Eds.), In: Mechanical Properties of Structural films, ASTM STP 1413, American Society for Testing and Materials, West Conshohocken, PA, 2001, 1.

[23] Tsuchiya, T. Fatigue Fract. Eng. Mater. Struct., 2005, 28, 665.

[24] Alan, T. Improving fracture properties of MEMS components by surface control, Ph.D. Dissertation, Cornell University, 2007.

[25] Lee, Y.; Tada, J.; Isono Y. Fatigue Fract. Eng. Mater. Struct., 2005, 28, 675 .

[26] Eisner, R.L. Acta Metall., 1955, 3(4), 414.

[27] Klein, M.; Hadrboletz, A.; Weiss, B.; Khatibi, G. Mater. Sci. Eng., 2001, A319-A321, 924.

[28] Connolley, T.; Mchugh, P.E.; Bruzzi, M. Fatigue Fract. Eng., Mater Struct., 2005, 28, 1119.

[29] Khatibi, G.; Betzwar-Kotas, A.; Groger, V.; Veiss, B. Fatigue Fract. Eng. Mater. Struct., 2005, 28, 723.

[30] Lou, J.; Allameh, S.; Buccheit, T.; Soboyejo, W.O. J. Mater. Sci., 2003, 38, 4129.

[31] Yang, Y.1 Imasogie, B.I.; Allameh, S.M.; Boyce, B.; Lian, K.; Lou, J.; Soboyejo, W.O. Mater. Sci. Eng., 2007, A444, 39.

[32] Fruhauf, J.; Gartner, E.; Jansch E. J. Micromech. Microeng., 1999, 9, 305 . 